UDC 594
A. E. Berezkina ${ }^{1}$, M. Yu. Shrestha ${ }^{2}$, O. I. Sinna ${ }^{2}$, D. V. Shmyrov ${ }^{3}$, A. Yu. Utevsky 1, 2, *
${ }^{1}$ State Institution National Antarctic Scientific Center, Ministry of Education and Science of Ukraine, 16 Taras Shevchenko Blvd., Kyiv, 01601, Ukraine
2 V. N. Karazin Kharkiv National University, 4 Svobody Sq., Kharkiv, 61022, Ukraine
${ }^{3}$ SITRONICS Telecom Solutions Ukraine, Ltd, bldg. 2d, 9 Stepana Bandery Ave, Kyiv, 04073, Ukraine
* Corresponding author: andriy.utevsky@karazin.ua

\title{
THE DISTRIBUTION OF THE ANTARCTIC LIMPET NACELLA CONCINNA (NACELLIDAE) ON UNDERWATER LANDSCAPES OF THE MEEK CHANNEL, ARGENTINE ISLANDS, GRAHAM LAND
}

\begin{abstract}
Objective(s). To clarify the patterns of Nacella concinna (Nacellidae) distribution in the water area of the Argentine Islands Archipelago (Meek Channel). Methods. Morphometric, statistical, cartographic methods as well as geoinformation technologies were used. Results. Analyzed the patterns of mollusc distribution on different transects, taking into account their morphometric characteristics - the length of the shell, the mollusc weight, the population density. It is determined that the distribution of Nacella concinna subpopulations does not have any visible strict regularity. At different transects with an insignificant exception the morphometric characteristics of the molluscs are distributed both in classical and non-classical models. At the MK1 transect is shown the classical model of the distribution of morphometric characteristics, namely with an increase in the mollusc population density, the morphometric characteristics (shell length and weight of the mollusc) tend to decrease. At the MK2 transect is shown non-classical distribution model. The morphometric characteristics (shell length, mollusc weight) increase with an increase in the population density of the molluscs. At the MK3 transect is shown the classical distribution model, but a characteristic feature is an increase in the population density up to $5 \mathrm{~m}$ depth and a gradual decrease in the population density from 5 to $20 \mathrm{~m}$. Geoinformation models of the Nacella concinna distribution by depth in the water area of the Ukrainian Antarctic Akademik Vernadsky station were constructed. Conclusions. Maps of the distribution of the Nacella concinna population were created for this region for the first time. The dimensional classes of the Nacella concinna subpopulation from selected water areas of the Argentine Islands were described.
\end{abstract}

Keywords: Nacella concinna, Meek Channel, Antarctic molluscs, West Antarctica, Argentine Islands.

\section{INTRODUCTION}

The Antarctic limpet Nacella concinna (Strebel, 1908) is a widespread species of gastropod mollusks in Antarctica. It could reach a significant density and abundance in some regions and areas of the sea floor (Walker, 1972; Picken, 1980; Picken and Allan, 1983; Peck and Veal, 2001). These mollusks have been found in a wide range of depths from the littoral zone to 110 meters (Picken, 1980; Davenport, 1988; Brethes et al., 1994).

(C) A. E. BEREZKINA, M. Yu. SHRESTHA, O. I. SINNA, D. V. SHMYROV, A. Yu. UTEVSKY, 2018

\section{2}

Their distribution by depth and underwater landscapes is related to the microalgae substrate richness, especially Lithothamnion species. Studies on the distribution of $N$. concinna by morphological types and depths have been undertaken by many authors in West Antarctica along the Antarctic Peninsula (Picken, 1980; Beaumont and Wei, 1991; Nolan, 1991; Gonzalez-Wevar, David, Poulin, 2011). Morphological and phylogeographical analyses of the mollusks have been conducted in the areas of South Bay, Elephant Island, Admiralty Bay, Fildes Bay and Covadonga Bay (Gonzalez-Wevar, David, Poulin, 2011). A population of N. concinna has been surveyed recen- 
tly near Anvers Island (Aranzamendi, Martinez, Sahade, 2010). A survey in these areas has revealed the existence of two morphotypes («littoral» and «sublittoral»).

Samplings of $N$. concinna were done during the period of 2003-2016 in the water area near the Vernadsky station (Utevsky, Tabikelova, Sennaya, Utevsky, 2015; Kushnir, Utevsky, 2015). The collected samples were used to deduce equations of the mollusk growth and to determine the correlation of the morphometric characteristics (Utevsky, Shrestha, Utevsky, 2017). The underwater polygon (Fig. 1) was established by A. Utevsky and D. Shmyrov in 2016 for studying the distribution of species by depths and landscapes. In this article we present results of the morphometric analysis of $N$. concinna and analysis of its distribution by depths and landscapes to test the hypothesis of the existence of littoral and sublittoral morphotypes.

\section{MATERIALS AND METHODS}

Three transects in the Meek Channel near Galindez Island in the summer season of 2016 were established (Fig. 1) and their base geocoordinates are given: MK1 -

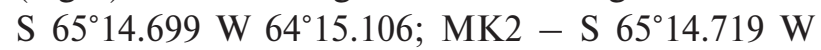
$64^{\circ} 15.023$, MK3 - S 65 $14{ }^{\prime} 42.73^{\prime \prime}$ W 64 15’4.90”.

The distance from the base of the transect MK1 to MK3 is approximately 29 meters, and from MK2 to MK3 - 33.9 meters. The base points location of transects was designated based on the relief of the above-water and underwater landscape. The diving method along transects with photographing and videotaping the characteristic features of the landscape and groups of benthic animals was used. Photographing of benthic animals at depths of $1 \mathrm{~m}, 5 \mathrm{~m}, 10 \mathrm{~m}, 15$ $\mathrm{m}, 20 \mathrm{~m}$, and $30 \mathrm{~m}$ using a standard square frame of $25 \times 25 \mathrm{~cm}$ was carried out. A series of photographs at each station was taken to quantify common species and calculate their morphometric parameters using VISION-ZEISS software package (Fig. 2, 3).

An acoustic survey was performed (Fig. 4) using a Lowrance HD7 chart plotter to reconstruct the bottom relief in the transect area.

The distribution pattern of $N$. concinna was obtained from underwater photographs (Table 1, Table 2).
The calculation of the biomass was performed using the equations (Utevsky et al., 2017):

for $\mathrm{L}<33 \mathrm{~mm} \mathrm{M}=0.0075 * \exp (0.2211 * \mathrm{~L})(1)$

for $\mathrm{L}>33 \mathrm{~mm} \mathrm{M}=0.4671 * \exp (0.0624 * \mathrm{~L})(2)$

The subsequent reconstruction of the sea bottom relief was carried out using ArcGIS ${ }^{\mathrm{Tm}}$ software package.

\section{RESULTS AND DISCUSSION}

Totally 225 mollusks from 3 transects and 11 underwater sites were analyzed. The descriptive statistics of $N$. concinna samples are presented in Table 1, Table 2 and Table 3. The distribution of mollusks along transects and stations by length of the shell and weight are shown in Fig. 5 and Fig. 6.

\section{Description of $N$. concinna population by transects}

Transect MK1. Shell length and mollusk weight decrease with population density increases (Table 1-3, Fig. 5, 6). It is consistent with classical ecological pattern in which an increase in a population density is accompanied by a decrease in the morphometric indices of organisms that usually associated with the lack of living space or food (Cohen, 2003). The highlevel correlation $(p<<0.05)$ of mollusk weight with population density confirms the inverse relationship. The high-level correlation of mollusk length with population density shows an inverse relationship of the parameters but statistically insignificant $(\mathrm{p}>0.05)$ which can be attributed to a small number of the samples (Fig. 7).

Transect MK2. Shell length and weight of the mollusk increase with population density increases (Table $1-3$, Fig. 5, 6). It is demonstrates a non-classical distribution of the population because morphometric characteristics of mollusks also increase with increasing of population density. Length of the shell increases with increasing of population density but the weight increases only slightly.

Dependence of mollusk weight and length on population density (Fig. 8) reflects a direct relation with the high-level of correlation $(r=0.98, r=0.93)$. However, in both cases correlation coefficient was statistically insignificant $(\mathrm{p}>0.05)$. Probably it is associated with a small number of the samples. 


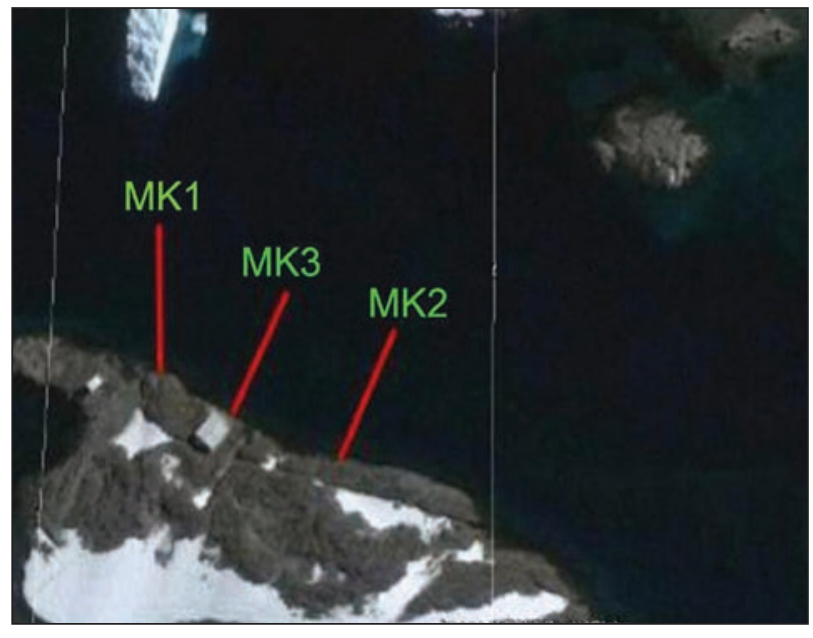

Fig. 1. Transects of an underwater polygon in the Meek Channel near Galindez Island: MK1, MK2, MK3
Transect MK3. Shell length and mollusk weight decrease with population density increases (Table $1-3$, Fig. 5, 6). It is consistent with the classical ecological pattern. Dependence of mollusk weight and length on population density reflects an inverse relation with the high-level of correlation $(r=0.80, r=$ $=0.95)$. But in both cases correlation coefficient was statistically insignificant ( $\mathrm{p}>0.05$ ) (Fig. 9).

\section{Description of $N$. concinna population by depths}

Depth of $1 \mathbf{m}$. The shell length from the MK1 transect has no significant differences from the shell length on the MK3 transect $(p=0.057)$. Similarly, the shell length on the MK3 and MK2 transect has no significant differences $(p=0.182)$. The mollusks from the
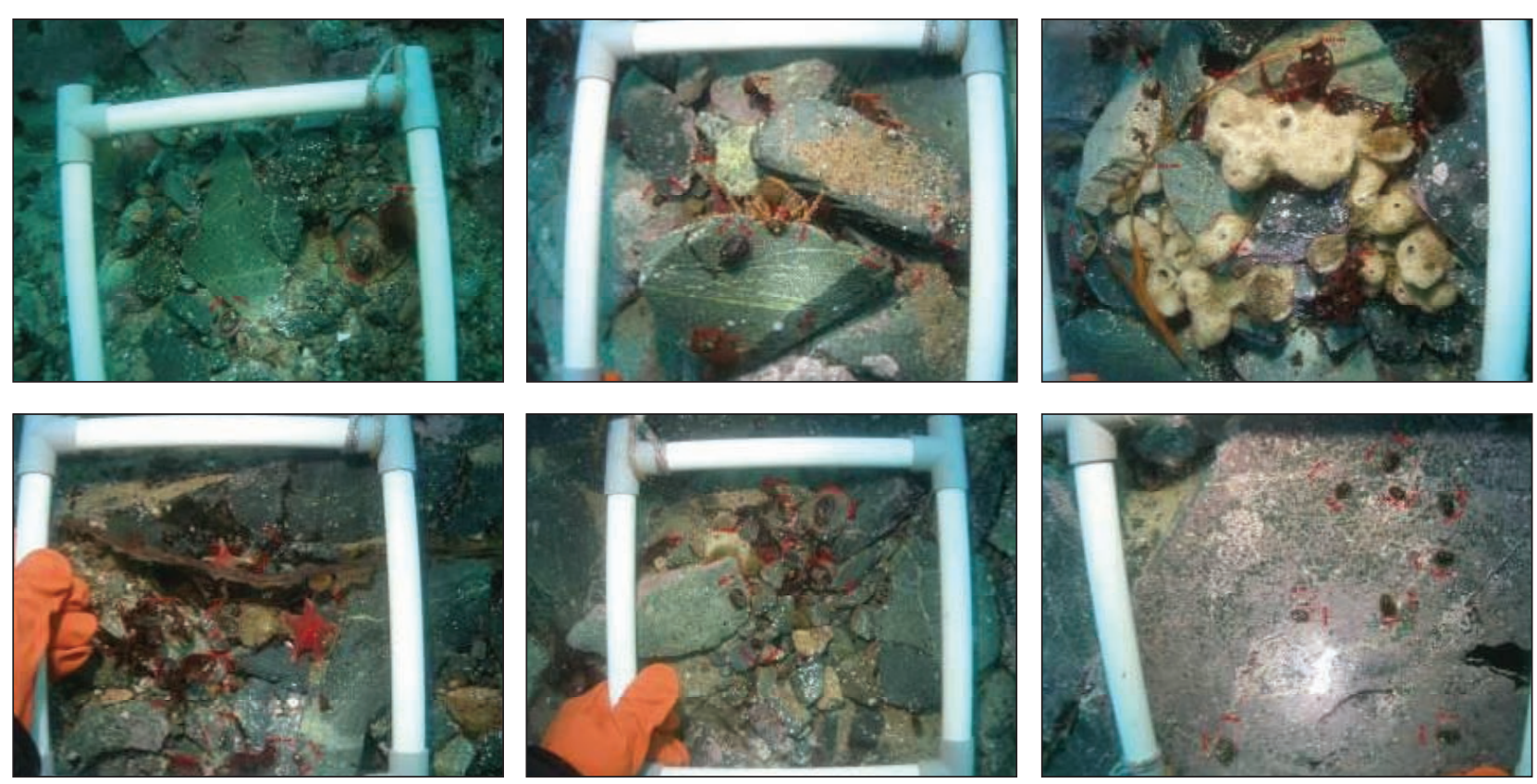

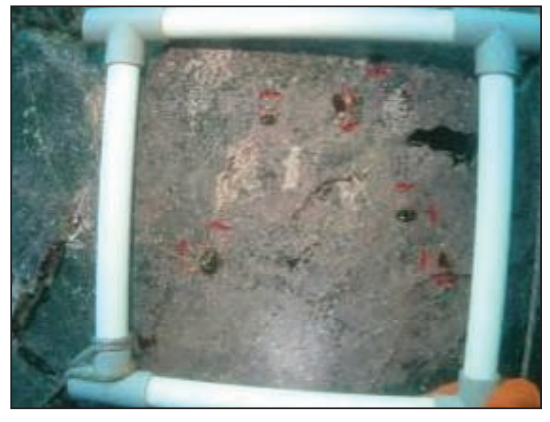

104
Fig. 2. Benthic communities of transect MK2, $5 \mathrm{~m}$ depth: diving method along transects using standard square frame $25 \times 25 \mathrm{~cm}$ 
MK2 transect do not differ in shell length from the mollusks from MK1 transect $(\mathrm{p}=0.663)$ (Table 4).

The mollusk weight at the $1 \mathrm{~m}$ depth does not have significant differences in all three transects. The weight of individuals from the MK1 transect do not differ from those in MK2 transect $(p=0.515)$. Also, the weight of individuals from the MK2 transect has no relevant differences from the weight of individuals from the MK3 transect $(p=0.140)$. Similarly, the weight from the MK3 and MK1 transect does not show differences $(\mathrm{p}=0.267)$ (Table 5).
Depth of $5 \mathrm{~m}$. A comparative analysis on $5 \mathrm{~m}$ depth shows statistically significant differences by shell length in all three transects compared with each other (Table 4). Thus the MK1 transect differs from MK2 transect, the MK2 from the MK3, the MK1 from the MK3 ( $p$ tends to " 0 ").

The weight of individuals from the MK1 transect compared with the weight of individuals from the MK2 transect has significant differences $(p=0.000)$. Also the weight from the MK1 transect has significant differences from the weight from the MK3 transect

Table 1. Comparative analysis by mean length $(\mathrm{L}, \mathrm{mm})$ of $N$. concinna along transects and depths

\begin{tabular}{|c|c|c|c|c|c|c|c|c|}
\hline $\begin{array}{c}\text { Transect } \\
\text { Parameter, mm }\end{array}$ & H, m & Valid N & Mean & Confidence Interval & Minimum & Maximum & Std. Dev. & Standard Err. \\
\hline MK-1L & 01 & 15 & 19.880 & $18.131-21.629$ & 12.000 & 25.100 & 3.158 & 0.816 \\
L & 05 & 15 & 24.113 & $21.790-26.436$ & 16.200 & 30.400 & 4.195 & 1.0831 \\
L & 10 & 4 & 26.125 & $13.435-38.815$ & 16.800 & 35.600 & 7.975 & 3.987 \\
MK-2L & 01 & 49 & 19.259 & $17.769-20.749$ & 10.700 & 34.400 & 5.187 & 0.741 \\
L & 05 & 36 & 16.861 & $15.211-18.512$ & 10.600 & 29.600 & 4.878 & 0.813 \\
L & 10 & 4 & 15.925 & $9.781-22.069$ & 12.100 & 21.300 & 3.861 & 1.931 \\
MK-3L & 01 & 23 & 17.643 & $16.091-19.196$ & 13.400 & 26.600 & 3.589 & 0.748 \\
L & 05 & 41 & 10.098 & $7.447-12.748$ & 2.400 & 32.100 & 8.398 & 1.312 \\
L & 10 & 10 & 11.260 & $4.173-18.347$ & 4.100 & 32.700 & 9.906 & 3.133 \\
L & 15 & 25 & 17.544 & $15.239-19.849$ & 4.500 & 30.500 & 5.583 & 1.117 \\
L & 20 & 3 & 30.733 & $1.772-59.695$ & 20.100 & 43.200 & 11.659 \\
\hline
\end{tabular}

Table 2. Comparative analysis by mean weight $(\mathrm{M}, \mathrm{g})$ of $N$. concinna along transects and depths

\begin{tabular}{|c|c|c|c|c|c|c|c|c|}
\hline $\begin{array}{c}\text { Transect } \\
\text { Parameter, g }\end{array}$ & H, m & Valid N & Mean & Confidence Interval & Minimum & Maximum & Std.Dev. & Standard Err. \\
\hline MK-1M & 01 & 15 & 0.742 & $0.481-1.002$ & 0.107 & 1.929 & 0.471 \\
M & 05 & 15 & 2.190 & $1.230-3.149$ & 0.270 & 6.225 & 1.733 & 0.122 \\
M & 10 & 4 & 6.390 & $-7.933-20.714$ & 0.308 & 19.655 & 9.001 & 4.501 \\
MK-2M & 01 & 49 & 0.976 & $0.587-1.365$ & 0.080 & 6.089 & 1.353 & 0.193 \\
M & 05 & 36 & 0.616 & $0.283-0.949$ & 0.078 & 5.216 & 0.983 \\
M & 10 & 4 & 0.342 & $-0.184-0.868$ & 0.109 & 0.83242 & 0.331 \\
MK-3M & 01 & 23 & 0.535 & $0.276-0.794$ & 0.145 & 2.687 & 0.599 \\
M & 05 & 41 & 0.372 & $0.107-0.638$ & 0.013 & 3.462 & 0.840 \\
M & 10 & 10 & 0.532 & $-0.281-1.346$ & 0.019 & 3.594 & 1.137 \\
M & 15 & 25 & 0.650 & $0.296-1.004$ & 0.020 & 3.133 & 0.125 \\
M & 20 & 3 & 3.465 & $-4.455-11.384$ & 0.638 & 6.920 & 3.188 \\
\hline
\end{tabular}




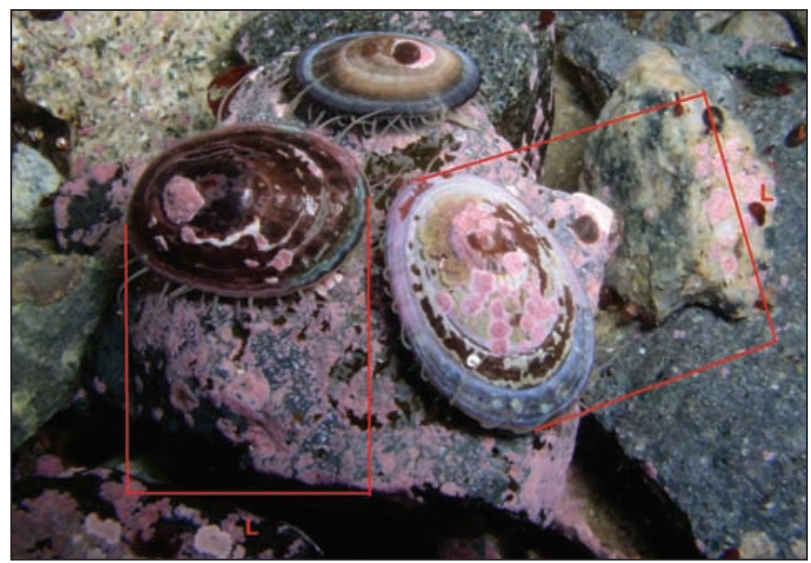

Fig. 3. Nacella concinna shell morphometry, VISION-ZEISS software package: L-length

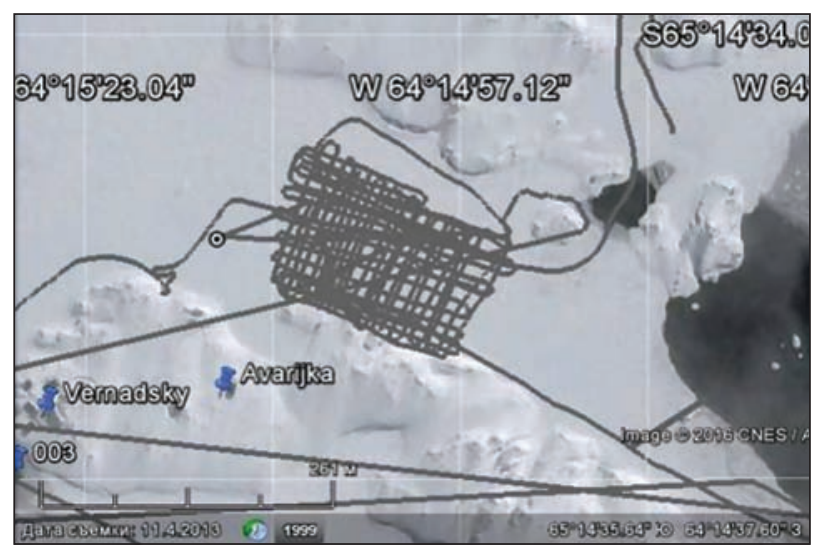

Fig. 4. Screenshot of acoustic survey track using a Lowrance HD7 chart plotter in the transect area

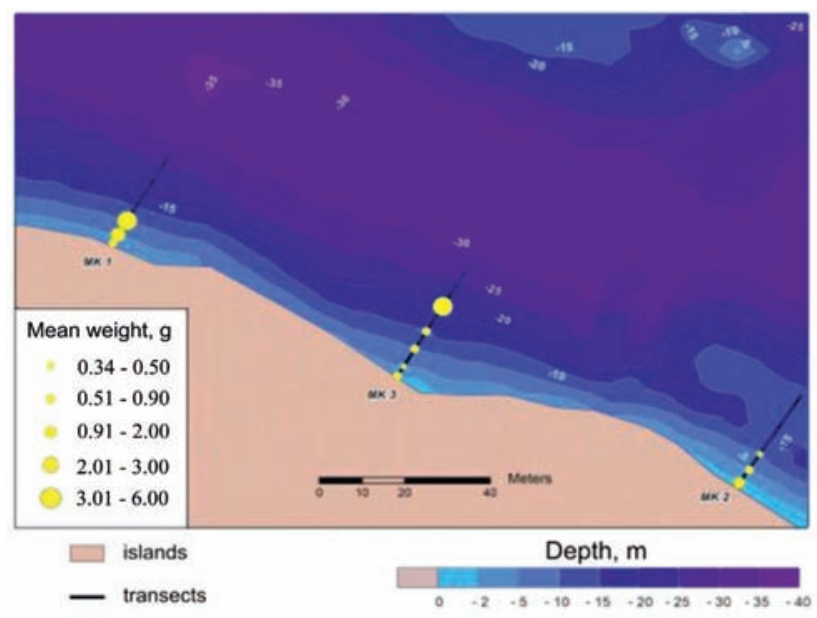

Fig. 5. Nacella concinna distribution by weight in Meek Channel transects using ArcGIS ${ }^{\mathrm{Tm}}$ software package

\section{6}

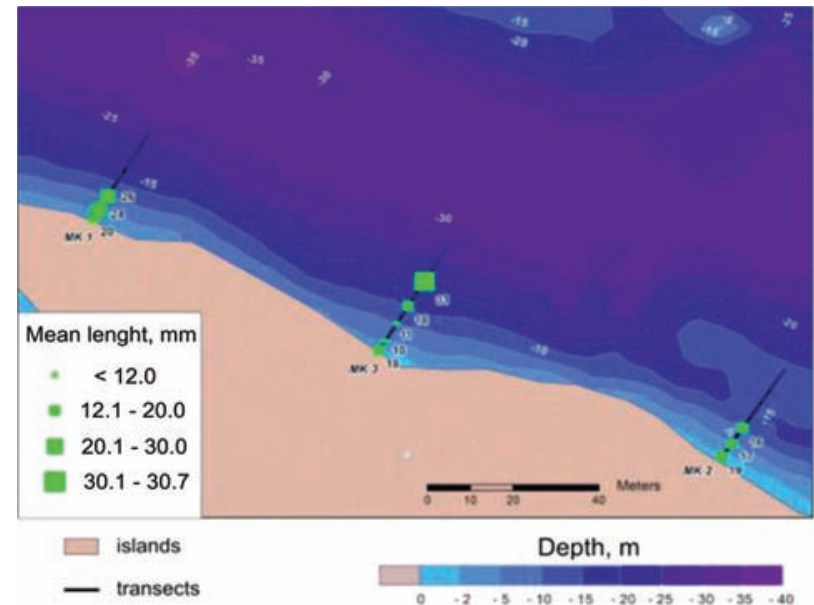

Fig. 6. Nacella concinna distribution by shell length in Meek Channel transects using ArcGIS ${ }^{\mathrm{Tm}}$ software package

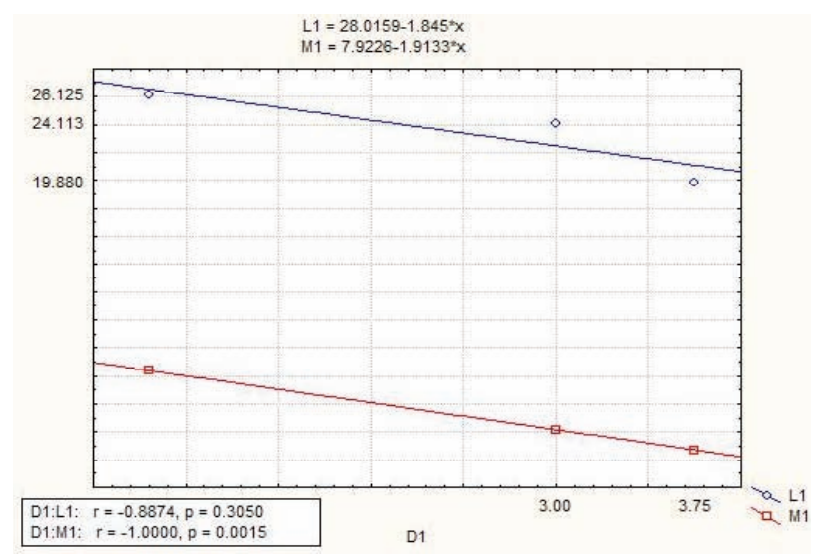

Fig. 7. Nacella concinna relation analyses of shell length (L1) and mollusk weight (M1) with population density (D1) on the MK1 transect

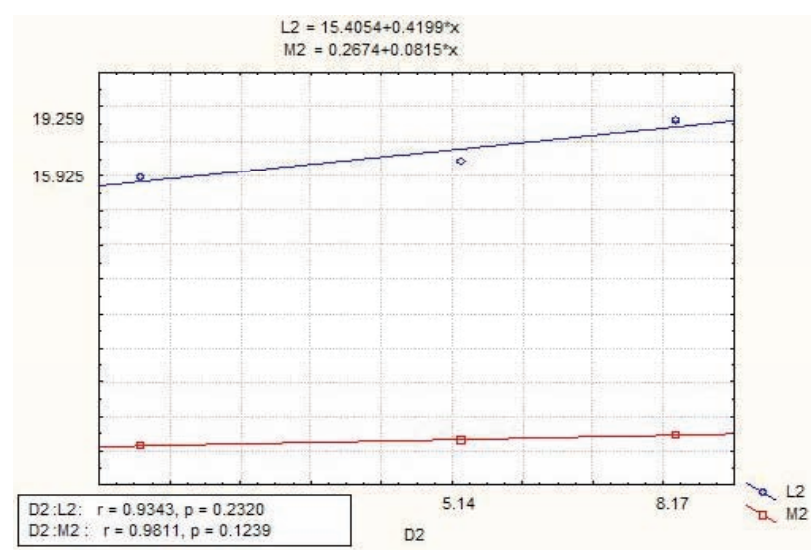

Fig. 8. Nacella concinna relation analyses of shell length (L2) and mollusk weight (M2) with population density (D2) on the MK2 transect

ISSN 1727-7485. Ukrainian Antarctic Journal. 2018, № 1(17) 
$(\mathrm{p}=0.000)$. At the same time the weight from the MK2 transect has no significant differences from the weight from the MK3 transect $(p=0.245)$ (Table 5).

Depth of $10 \mathrm{~m}$. A comparative analysis on $10 \mathrm{~m}$ depth shows statistically significant differences by shell length only between the MK1 and MK2 ( $p=0.061)$ and between the MK2 the MK3 transects $(p=0.388)$ compared with each other. The shell length from the MK1 transect has significant differences from the shell length on the MK3 transect ( $p=0.021)$ (Table 4).

The weight of individuals from the MK1 transect compared with MK2 transect has no significant differences $(p=0.228)$. Also, the weight from the MK2 transect does not differ from the weight from the MK3 transect $(p=0.753)$. However, the weight of individuals from the MK3 transect has significant differences from the weight of individuals from the MK1 transect $(\mathrm{p}=0.053)$ (Table 5).

Shell weight and length of the $N$. concinna increase at depth from 1 to $10 \mathrm{~m}$ on the MK1 transect. This is a classical model of the biomass chain where a small number of individuals in an ecotope with a sufficient amount of food are characterized by large morphometric indices (Cohen, 2003). The direct dependence of the increase in nutrient substrate (algae of the genus Lithothamnion) with increasing depth is visually observed on the MK1 transect.

On the MK2 transect the shell length decreases at the depth from 1 to $10 \mathrm{~m}$. The high-level correlation of the shell length, weight, population density with depth are shows an inverse dependence. The correlation of the depth, weight and density are confirmed

Table 3. Population density (mollusk quantity per frame) of $N$. concinna on transects

\begin{tabular}{|c|c|c|c|c|c|c|c|c|c|c|c|c|c|c|c|}
\hline \multirow{3}{*}{ Transects } & \multicolumn{15}{|c|}{ Depth (m) } \\
\hline & \multicolumn{3}{|c|}{1} & \multicolumn{3}{|c|}{5} & \multicolumn{3}{|c|}{10} & \multicolumn{3}{|c|}{15} & \multicolumn{3}{|c|}{20} \\
\hline & 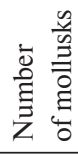 & 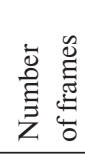 & 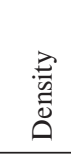 & 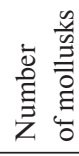 & 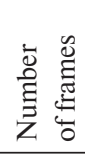 & 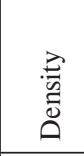 & 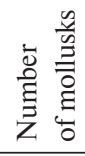 & 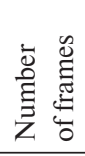 & $\begin{array}{l}\overrightarrow{0} \\
\text { D. } \\
\stackrel{0}{0} \\
\end{array}$ & 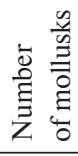 & 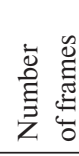 & $\begin{array}{l}\overrightarrow{2} \\
\stackrel{0}{0} \\
\stackrel{0}{0} \\
\end{array}$ & 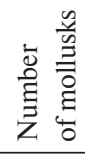 & 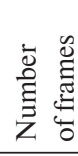 & 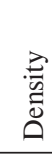 \\
\hline MK-1 & 15 & 4 & 3.75 & 15 & 5 & 3 & 4 & 5 & 0.8 & - & 5 & - & - & 1 & - \\
\hline MK-2 & 49 & 6 & 8.17 & 36 & 7 & 5.14 & 4 & 7 & 0.57 & - & 8 & - & - & 7 & - \\
\hline MK-3 & 23 & 4 & 5.75 & 41 & 3 & 13.67 & 10 & 1 & 10 & 25 & 4 & 6.25 & 3 & 6 & 0.5 \\
\hline
\end{tabular}

Table 4. Comparison of $N$. concinna by length at different transect horizons

\begin{tabular}{|l|c|c|c|c|c|c|c|c|}
\hline \multicolumn{1}{|c|}{ Parameter } & Mean & Mean & P & Valid N & Valid N & Std. Dev. & Std. Dev. & p \\
\hline L 3-1 vs. L 2-1 & 17.643 & 19.259 & 0.182 & 23.000 & 49.000 & 3.589 & 5.187 & 0.063 \\
L 3-1 vs. L 2-5 & 17.643 & 16.861 & 0.510 & 23.000 & 36.000 & 3.589 & 4.878 & 0.132 \\
L 3-1 vs. L 2-10 & 17.643 & 15.925 & 0.390 & 23.000 & 4.000 & 3.589 & 3.861 & 0.697 \\
L 3-1 vs. L 1-1 & 17.643 & 19.880 & 0.057 & 23.000 & 15.000 & 3.589 & 3.158 & 0.631 \\
L 3-1 vs. L 1-5 & 17.643 & 24.113 & 0.000 & 23.000 & 15.000 & 3.589 & 4.195 & 0.498 \\
L 3-1 vs. L 1-10 & 17.643 & 26.125 & 0.001 & 23.000 & 4.000 & 3.589 & 7.975 & 0.018 \\
L 3-5 vs. L 2-1 & 10.098 & 19.259 & 0.000 & 41.000 & 49.000 & 8.398 & 5.187 & 0.002 \\
L 3-5 vs. L 2-5 & 10.098 & 16.861 & 0.000 & 41.000 & 36.000 & 8.398 & 4.878 & 0.001 \\
L 3-5 vs. L 2-10 & 10.098 & 15.925 & 0.180 & 41.000 & 4.000 & 8.398 & 3.861 & 0.224 \\
L 3-5 vs. L 1-1 & 10.098 & 19.880 & 0.000 & 41.000 & 15.000 & 8.398 & 3.158 & 0.000
\end{tabular}


End of Table 4

\begin{tabular}{|c|c|c|c|c|c|c|c|c|}
\hline Parameter & Mean & Mean & $\mathrm{P}$ & Valid N & Valid $N$ & Std. Dev. & Std. Dev. & $\mathrm{p}$ \\
\hline L 3-5 vs. L 1-5 & 10.098 & 24.113 & 0.000 & 41.000 & 15.000 & 8.398 & 4.195 & 0.007 \\
\hline L 3-5 vs. L 1-10 & 10.098 & 26.125 & 0.001 & 41.000 & 4.000 & 8.398 & 7.975 & 1.000 \\
\hline L 3-10 vs. L 2-1 & 11.260 & 19.259 & 0.000 & 10.000 & 49.000 & 9.907 & 5.187 & 0.003 \\
\hline L $3-10$ vs. L 2-5 & 11.260 & 16.861 & 0.016 & 10.000 & 36.000 & 9.907 & 4.878 & 0.002 \\
\hline L 3-10 vs. L 2-10 & 11.260 & 15.925 & 0.388 & 10.000 & 4.000 & 9.907 & 3.861 & 0.148 \\
\hline L 3-10 vs. L 1-1 & 11.260 & 19.880 & 0.004 & 10.000 & 15.000 & 9.907 & 3.158 & 0.000 \\
\hline L 3-10 vs. L 1-5 & 11.260 & 24.113 & 0.000 & 10.000 & 15.000 & 9.907 & 4.195 & 0.005 \\
\hline L $3-10$ vs. L $1-10$ & 11.260 & 26.125 & 0.021 & 10.000 & 4.000 & 9.907 & 7.975 & 0.793 \\
\hline L 3-15 vs. L 2-1 & 17.544 & 19.259 & 0.194 & 25.000 & 49.000 & 5.583 & 5.187 & 0.648 \\
\hline L 3-15 vs. L 2-5 & 17.544 & 16.861 & 0.614 & 25.000 & 36.000 & 5.583 & 4.878 & 0.458 \\
\hline L 3-15 vs. L 2-10 & 17.544 & 15.925 & 0.584 & 25.000 & 4.000 & 5.583 & 3.861 & 0.599 \\
\hline L 3-15 vs. L 1-1 & 17.544 & 19.880 & 0.147 & 25.000 & 15.000 & 5.583 & 3.158 & 0.031 \\
\hline L 3-15 vs. L 1-5 & 17.544 & 24.113 & 0.000 & 25.000 & 15.000 & 5.583 & 4.195 & 0.268 \\
\hline L 3-15 vs. L 1-10 & 17.544 & 26.125 & 0.012 & 25.000 & 4.000 & 5.583 & 7.975 & 0.270 \\
\hline L 3-20 vs. L 2-1 & 30.733 & 19.259 & 0.001 & 3.000 & 49.000 & 11.659 & 5.187 & 0.020 \\
\hline L 3-20 vs. L 2-5 & 30.733 & 16.861 & 0.000 & 3.000 & 36.000 & 11.659 & 4.878 & 0.014 \\
\hline L 3-20 vs. L 2-10 & 30.733 & 15.925 & 0.059 & 3.000 & 4.000 & 11.659 & 3.861 & 0.106 \\
\hline L 3-20 vs. L 1-1 & 30.733 & 19.880 & 0.004 & 3.000 & 15.000 & 11.659 & 3.158 & 0.001 \\
\hline L 3-20 vs. L 1-5 & 30.733 & 24.113 & 0.085 & 3.000 & 15.000 & 11.659 & 4.195 & 0.011 \\
\hline L 3-20 vs. L $1-10$ & 30.733 & 26.125 & 0.558 & 3.000 & 4.000 & 11.659 & 7.975 & 0.530 \\
\hline L 2-1 vs. L 1-1 & 19.259 & 19.880 & 0.663 & 49.000 & 15.000 & 5.187 & 3.158 & 0.046 \\
\hline L 2-1 vs. L 1-5 & 19.259 & 24.113 & 0.002 & 49.000 & 15.000 & 5.187 & 4.195 & 0.389 \\
\hline L 2-1 vs. L 1-10 & 19.259 & 26.125 & 0.018 & 49.000 & 4.000 & 5.187 & 7.975 & 0.166 \\
\hline L 2-5 vs. L 1-1 & 16.861 & 19.880 & 0.032 & 36.000 & 15.000 & 4.878 & 3.158 & 0.084 \\
\hline L 2-5 vs. L $1-5$ & 16.861 & 24.113 & 0.000 & 36.000 & 15.000 & 4.878 & 4.195 & 0.557 \\
\hline L $2-5$ vs. L $1-10$ & 16.861 & 26.125 & 0.002 & 36.000 & 4.000 & 4.878 & 7.975 & 0.125 \\
\hline L $2-10$ vs. L 1-1 & 15.925 & 19.880 & 0.048 & 4.000 & 15.000 & 3.861 & 3.158 & 0.518 \\
\hline L 2-10 vs. L 1-5 & 15.925 & 24.113 & 0.003 & 4.000 & 15.000 & 3.861 & 4.195 & 1.000 \\
\hline L $2-10$ vs. L $1-10$ & 15.925 & 26.125 & 0.061 & 4.000 & 4.000 & 3.861 & 7.975 & 0.264 \\
\hline
\end{tabular}

Note: the significant differences are grey $(p<0.05)$. 
Table 5. Comparison of $N$. concinna by weight at different transect horizons

\begin{tabular}{|c|c|c|c|c|c|c|c|c|}
\hline M 3-1 vs. M 2-1 & 0.535 & 0.976 & 0.140 & 23.000 & 49.000 & 0.599 & 1.353 & 0.000 \\
\hline M 3-1 vs. M 2-10 & 0.535 & 0.342 & 0.540 & 23.000 & 4.000 & 0.599 & 0.331 & 0.356 \\
\hline M 3-1 vs. M 1-1 & 0.535 & 0.742 & 0.267 & 23.000 & 15.000 & 0.599 & 0.471 & 0.355 \\
\hline M 3-5 vs. M 2-1 & 0.372 & 0.976 & 0.015 & 41.000 & 49.000 & 0.840 & 1.353 & 0.002 \\
\hline M 3-5 vs. M 2-5 & 0.372 & 0.616 & 0.245 & 41.000 & 36.000 & 0.840 & 0.983 & 0.336 \\
\hline M 3-5 vs. M 2-10 & 0.372 & 0.342 & 0.944 & 41.000 & 4.000 & 0.840 & 0.331 & 0.148 \\
\hline M 3-5 vs. M 1-1 & 0.372 & 0.742 & 0.114 & 41.000 & 15.000 & 0.840 & 0.471 & 0.022 \\
\hline M 3-10 vs. M 2-1 & 0.532 & 0.976 & 0.337 & 10.000 & 49.000 & 1.137 & 1.353 & 0.600 \\
\hline M 3-10 vs. M 2-5 & 0.532 & 0.616 & 0.819 & 10.000 & 36.000 & 1.137 & 0.983 & 0.507 \\
\hline M 3-10 vs. M 2-10 & 0.532 & 0.342 & 0.753 & 10.000 & 4.000 & 1.137 & 0.331 & 0.066 \\
\hline M 3-10 vs. M 1-1 & 0.532 & 0.742 & 0.528 & 10.000 & 15.000 & 1.137 & 0.471 & 0.004 \\
\hline M 3-10 vs. M 1-5 & 0.532 & 2.190 & 0.014 & 10.000 & 15.000 & 1.137 & 1.733 & 0.207 \\
\hline M 3-10 vs. M 1-10 & 0.532 & 6.390 & 0.053 & 10.000 & 4.000 & 1.137 & 9.001 & 0.000 \\
\hline M 3-15 vs. M 2-1 & 0.650 & 0.976 & 0.276 & 25.000 & 49.000 & 0.857 & 1.353 & 0.018 \\
\hline M 3-15 vs. M 2-5 & 0.650 & 0.616 & 0.891 & 25.000 & 36.000 & 0.857 & 0.983 & 0.489 \\
\hline M 3-15 vs. M 2-10 & 0.650 & 0.342 & 0.490 & 25.000 & 4.000 & 0.857 & 0.331 & 0.141 \\
\hline M 3-20 vs. M 2-10 & 3.465 & 0.342 & 0.100 & 3.000 & 4.000 & 3.188 & 0.331 & 0.004 \\
\hline M 3-20 vs. M 1-1 & 3.465 & 0.742 & 0.003 & 3.000 & 15.000 & 3.188 & 0.471 & 0.000 \\
\hline M 3-20 vs. M 1-5 & 3.465 & 2.190 & 0.322 & 3.000 & 15.000 & 3.188 & 1.733 & 0.126 \\
\hline M 3-20 vs. M 1-10 & 3.465 & 6.390 & 0.620 & 3.000 & 4.000 & 3.188 & 9.001 & 0.227 \\
\hline M 2-1 vs. M 1-1 & 0.976 & 0.742 & 0.515 & 49.000 & 15.000 & 1.353 & 0.471 & 0.000 \\
\hline M 2-1 vs. M 1-5 & 0.976 & 2.190 & 0.006 & 49.000 & 15.000 & 1.353 & 1.733 & 0.205 \\
\hline M 2-1 vs. M 1-10 & 0.976 & 6.390 & 0.000 & 49.000 & 4.000 & 1.353 & 9.001 & 0.000 \\
\hline M 2-5 vs. M 1-1 & 0.616 & 0.742 & 0.640 & 36.000 & 15.000 & 0.983 & 0.471 & 0.005 \\
\hline M 2-5 vs. M 1-5 & 0.616 & 2.190 & 0.000 & 36.000 & 15.000 & 0.983 & 1.733 & 0.007 \\
\hline M 2-5 vs. M 1-10 & 0.616 & 6.390 & 0.000 & 36.000 & 4.000 & 0.983 & 9.001 & 0.000 \\
\hline M 2-10 vs. M 1-1 & 0.342 & 0.742 & 0.132 & 4.000 & 15.000 & 0.331 & 0.471 & 0.615 \\
\hline M 2-10 vs. M 1-5 & 0.342 & 2.190 & 0.053 & 4.000 & 15.000 & 0.331 & 1.733 & 0.019 \\
\hline M 2-10 vs. M 1-10 & 0.342 & 6.390 & 0.228 & 4.000 & 4.000 & 0.331 & 9.001 & 0.000 \\
\hline
\end{tabular}

Note: the significant differences are grey $(p<0.05)$. 


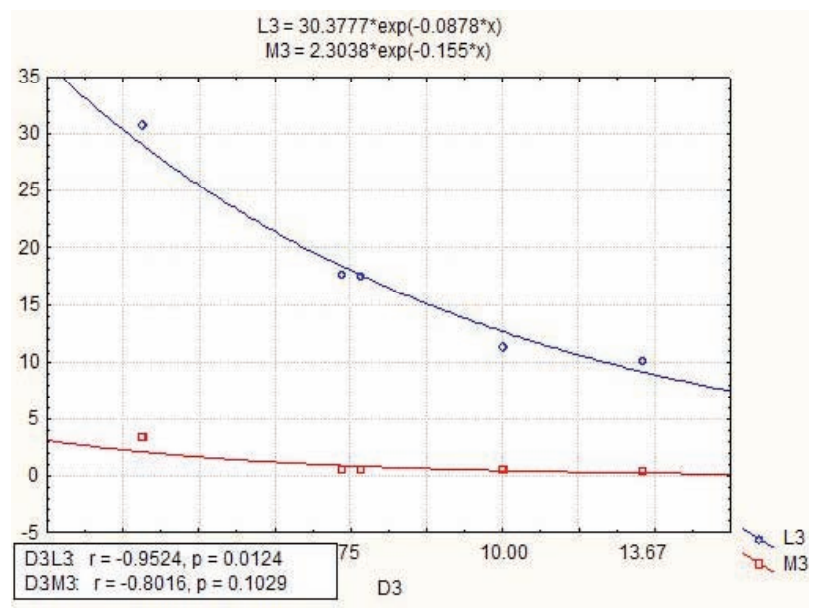

Fig. 9. Nacella concinna relation analyses of shell length (L3) and mollusk weight (M3) with population density (D3) on the MK3 transect

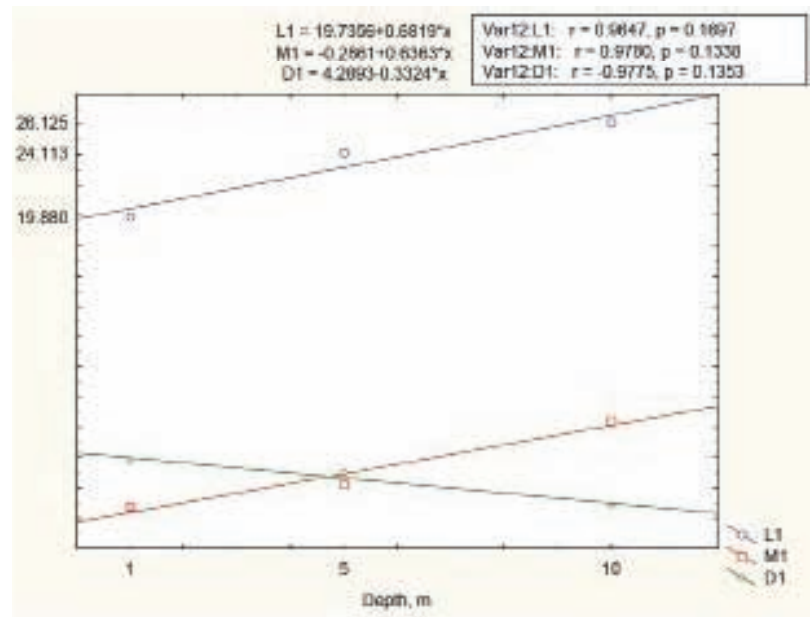

Fig. 10. Nacella concinna relation analyses of length (L1), weight (M1) and population density (D1) with depth on the MK1 transect

$(\mathrm{p}<0.05)$. The correlation of the depth and length are not confirmed $(\mathrm{p}>0.05)$. The mollusk weight shows a slight tendency to decreases as the sharp decreases of the population density (Fig. 11). A non-classical distribution model is observed on the MK2 transect: density of the population and the morphometric parameters (length and weight) decrease by depth increasing. But by visual observing the nutrition availability at a depth of $10 \mathrm{~m}$ is more than sufficient.

On the MK3 transect the classical model of distribution of the $N$. concinna population was deter-

\section{0}

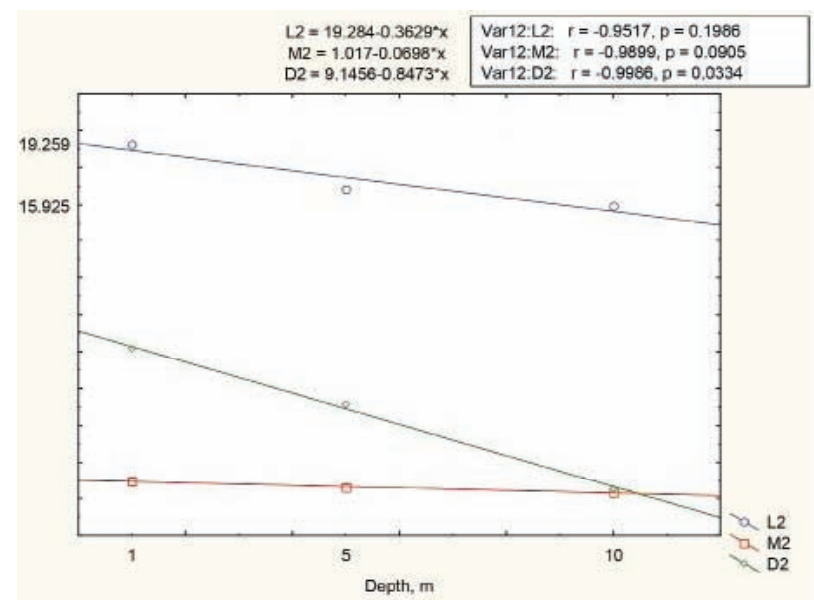

Fig. 11. Nacella concinna relation analyses of length (L2), weight (M2) and population density (D2) with depth on the MK2 transect

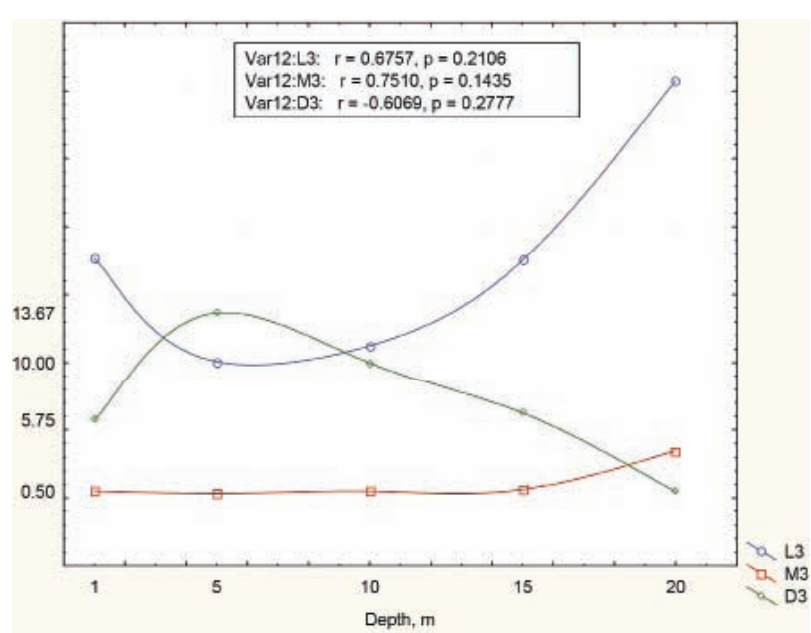

Fig. 12. Nacella concinna relation analyses of length (L3), weight (M3) and population density (D3) with depth on the MK3 transect

mined. The high-level correlation of the shell length, weight, population density and depth are not confirmed ( $p>0.05)$ The mollusk weight increases at depth from 1 to $20 \mathrm{~m}$ and shell length increases only from $6 \mathrm{~m}$ depth.

Population density varies by depth: increases at the $5 \mathrm{~m}$, gradually decreases deeper up to $20 \mathrm{~m}$ (Fig. 12). Such the weight, length and population density distribution on this transect could be related to nutrition access at a depth of up to $5 \mathrm{~m}$ and after $15 \mathrm{~m}$, wave influence and underwater landscapes structure.

ISSN 1727-7485. Ukrainian Antarctic Journal. 2018, № 1(17) 


\section{CONCLUSIONS}

An obvious correlation of the mollusk shell length with the depth on the transects MK1, MK2, MK3 was not observed. The complex relations of the $N$. concinna shell weight, length and distribution by depth on the transects MK1, MK2, MK3 does not correspond to previous literature records. The separation of the mollusk population into littoral and sublittoral morphotypes was not confirmed for surveyed Meek Channel water area in the Argentine Islands. Apparently, the shell morphology and the weight of $N$. concinna depend on the bottom relief, nutrition access (abundance of algae) and wave activity on each site of transects under study.

Acknowledgments. The study was supported by the grant from the President of Ukraine (project F70/1372017 of the State Fund for Fundamental Research (No. of the SRW 0117U003557); the National Antarctic Scientific Center of the Ministry of Education and Science of Ukraine, the project N/17-2017, No. 011UA007599).

\section{REFERENCES}

1. Aranzamendi, M. C., Martinez, J. J., Sahade, R. 2010. Shape differentiation and characterization in the two morphotypes of the Antarctic limpet Nacella concinna using Elliptic Fourier analysis of shells. Polar Biology, 33, 1163-1170. doi:10.1007/s00300-010-0803-2.

2. Beaumont, A. R., Wei, J. H. C. 1991. Morphological and genetic variation in the Antarctic limpet Nacella concinna. Journal of Molluscan Studies, 57, 443-450.

3. Brethes, J.-C., Ferreyra, G., Vega, S. 1994. Distribution, growth and reproduction of the limpet Nacella (Patinigera) concinna (Strebel, 1908) in relation to potential food availability, in Esperanza Bay (Antarctic Peninsula). Polar Biology, 14, 161-170.

4. Cohen, J. E., Jonsson, T., Carpenter S. R. 2003. Ecological community description using the food web, species abundance, and body size. Proceedings of the National
Academy of Sciences, 100 (4), 1781-1786; DOI:10.1073/ pnas. 232715699.

5. Davenport, J. 1988. Tenacity of the Antarctic limpet $\mathrm{Na}$ cella concinna. Journal of Molluscan Studies, 54, 355356.

6. Gonzalez-Wevar, C. A., David, B., Poulin, E. 2011. Phylogeography and demographic inference in Nacella (Patinigera) concinna (Strebel, 1908) in the western Antarctic Peninsula. Deep Sea Research Part II: Topical Studies in Oceanography, 58, 220-229. DOI:10.1016/j.dsr2.2010.05.026.

7. Kushnir, M. A., Utevsky, A. Y. 2015. Morphological diversity of the Antarctic limpet Nacella concinna (Gastropoda: Nacellidae) in the waters of the Argentine islands. Antarctic Research: New Horizons and Priorities (VII International Antarctic Conference). Kyiv, 12-14 May, 2015, 63-64.

8. Nolan, C. P. 1991. Size, shape and shell morphology in the Antarctic limpet Nacella concinna at Signy Island, South Orkney Islands. Journal of Molluscan Studies, 57, 225-238.

9. Picken, G. B. 1980. The distribution, growth, and reproduction of the Antarctic limpet Nacella (Patinigera) concinna (Strebel, 1908). Journal of Experimental Marine Biology and Ecology, 42, 71-85.

10. Picken, G. B., Allan, D. 1983. Unique spawning behaviour by the Antarctic limpet Nacella (Patinigera) concinna (Strebel 1908). Journal of Experimental Marine Biology and Ecology, 71, 283-287.

11. Peck, L., Veal, R. 2001. Feeding, metabolism and growth in the Antarctic limpet, Nacella concinna (Strebel, 1908). Marine Biology Journal, 138, 553-560.

12. Utevsky, A.Yu., Tabikelova, K.S., Sennaya, E.I., Utevsky, S.Yu. 2015. Changes of population structure in common benthic species of the proposed Stella Creek MPA in the vicinity of the Akademik Vernadsky Station, Galindez Island, Antarctica. CCAMLR Science: EMM-15-41.

13. Utevsky, A.Yu., Shrestha, M.Yu., Utevsky, S.Yu. 2017. Modelling the morphological features correlations of the $\mathrm{Na}$ cella concina (Strebel, 1908) (Patellogastropoda; Nacellidae) for the analysis of the benthic landscapes images. VIII International Antarctic Conference dedicated to the $24^{\text {th }}$ Anniversary of Ukraine's accession to the Antarctic Treaty. Kyiv, 16-18 May, 2017, 103-105.

14. Walker, A. J. M. 1972. Introduction to the ecology of the Antarctic limpet Patinigera polaris (Hombron and Jacquinot) at Signy Island, South Orkney Islands. British Antarctic Survey Bulletin, 28, 49-69. 
А.Є. Березкіна ${ }^{1}$, М.Ю. Шрестха², О.І. Сінна², Д.В. Шмирьов ${ }^{3}$, А.Ю. Утєвський

${ }^{1}$ Державна установа Національний антарктичний науковий центр МОН України, м. Київ

${ }^{2}$ Харківський національний університет імені В. Н. Каразіна, м. Харків

${ }^{3}$ ТОВ «СІТРОНІКС ТЕЛЕКОМ СОЛЮШНС УКРАЇНА», м. Київ

* Corresponding author: andriy.utevsky@karazin.ua

РОЗПОДІЛ АНТАРКТИЧНОЇ ЧАШЕЧКИ NACELLA CONCINNA (NACELLIDAE) НА ПІДВОДНИХ ЛАНДШАФТАХ ПРОТОКИ МІІ, АРГЕНТИНСЬКІ ОСТРОВИ, ЗЕМЛЯ ГРЕЙАМА

РЕФЕРАТ. Мета. 3'ясувати закономірності розподілу молюска Nacella concinna (Nacellidae) в акваторії архіпелагу Apгентинські острови (протока Міік). Методи. Використані морфометричні, статистичні, картографічні методи, а також геоінформаційні технології. Результати. Проаналізовано закономірності розподілу молюсків на різних трансектах з урахуванням їх морфометричних характеристик - довжини раковини, ваги молюска, щільності поселення. Встановлено, що розподіл субпопуляцій Nacella concinna не має будь-яких видимих строгих закономірностей. На різних трансектах на незначному віддаленні, морфометричні характеристики молюсків у вибірках розподіляються як за класичними, так і не за класичними моделями. Для трансекти MK1 показана класична модель розподілу морфометричних характеристик - зі збільшенням щільності поселення молюска морфометричні характеристики молюсків (довжина раковини і вага молюска) мають тенденцію до зменшення. Для трансекти МК2 показана некласична модель розподілу, де зі збільшенням щільності поселення молюска морфометричні характеристики молюсків (довжина раковини, вага особини) також збільшуються. Для трансекти МК3 також показана класична модель розподілу, але характерною особливістю є збільшення щільності поселення молюска до 5 метрів глибини і поступове зниження щільності поселення до глибини 20 м. Побудовані геоінформаційні моделі розподілу популяції Nacella concinna за глибинами в акваторії Української антарктичної станції «Академік Вернадський». Висновки. Вперше для зазначеного регіону створено карти розподілу популяції молюска Nacella concinna, а також описані окремі субпопуляції з їх розмірними класами в акваторії архіпелагу Аргентинські острови.

Ключові слова: Nacella concinna, протока Міік, антарктичні молюски, Західна Антарктика, Аргентинські острови. 